\title{
BILEVEL PROGRAMS WITH EXTREMAL VALUE FUNCTION: GLOBAL OPTIMALITY
}

\author{
ABDELMALEK ABOUSSOROR, HICHAM BABAHADDA, \\ AND ABDELATIF MANSOURI
}

Received 4 May 2004 and in revised form 29 October 2004

For a bilevel program with extremal value function, a necessary and sufficient condition for global optimality is given, which reduces the bilevel program to a max-min problem with linked constraints. Also, for the case where the extremal value function is polyhedral, this optimality condition gives the possibility of a resolution via a maximization problem of a polyhedral convex function over a convex set. Finally, this case is completed by an algorithm.

\section{Introduction}

We consider the following bilevel programming problem:

$(S)$

$$
\min _{\substack{x \in \mathbb{R}^{n} \\ G(x) \leq 0}} F(x, v(x))
$$

where $v(x)$ is the minimal value of the lower-level problem

$$
(P(x))
$$

$$
\min _{\substack{y \in \mathbb{R}^{m} \\ g(x, y) \leq 0}} f(x, y)
$$

and

$$
\begin{gathered}
F: \mathbb{R}^{n} \times \mathbb{R} \longrightarrow \mathbb{R}, \quad G=\left(G_{1}, \ldots, G_{p}\right): \mathbb{R}^{n} \longrightarrow \mathbb{R}^{p}, \\
f: \mathbb{R}^{n} \times \mathbb{R}^{m} \longrightarrow \mathbb{R}, \quad g=\left(g_{1}, \ldots, g_{q}\right): \mathbb{R}^{n} \times \mathbb{R}^{m} \longrightarrow \mathbb{R}^{q},
\end{gathered}
$$

are convex functions. Set

$$
\mathscr{G}=\left\{x \in \mathbb{R}^{n} / G(x) \leq 0\right\}, \quad Y(x)=\left\{y \in \mathbb{R}^{m} / g(x, y) \leq 0\right\} .
$$


The problem $(S)$ which is called a bilevel program with extremal value function, corresponds to a two-player game where a leader plays against a follower. The leader with the objective function $F$ chooses first a strategy $x \in \mathscr{G}$, and then the follower with the objective function $f$ reacts optimally by choosing $y \in Y(x)$. It is assumed that the leader evaluates the performance of the follower by his minimal value $v(x)$. Hence, he includes it in his objective function $F$. A more general formulation is considered in $[6,12,13]$, where the leader includes $v(x)$ in the objective and the constraint functions. Many optimization problems arising in practice can be classified into the type of $(S)$, we quote, for example, the two-level design problem and the general resource allocation problem for a decentralized system (for more details and examples we refer to [13]). As is well known, the marginal function $v$ is not differentiable in general, and hence problem $(S)$ requires nondifferentiable optimization techniques to be solved. We mention some recent works in this topic. In [14], for problem $(S)$, Tanino and Ogawa have proposed an algorithm based on the subgradient formula for the leader's objective function. As mentioned above, in $[6,12,13]$ the authors have considered a bilevel program $(\widetilde{S})$ in which the objective and the constraint functions include the marginal function $v$. In [6], Ishizuka was interested in finding optimality conditions for quasidifferentiable programs, and in particular, he provided an optimality condition for $(\widetilde{S})$. In $[12,13]$, using the directional derivatives of the function $v$, the authors have given optimality conditions for $(\widetilde{S})$, and proposed a computational method. In this paper, based essentially on the study given by Tuy in [18] for reverse convex programs, we provide a necessary and sufficient condition for global optimality. This condition opens another way for a possible resolution via minmax problems with linked constraints. For papers dealing with max-min solutions in the linked constraints case, we refer, for example, to $[5,7,10,11]$. Also, we consider the case where the function $f$ is polyhedral and $g$ is linear. In this case, the optimality condition offers another possibility of resolution of $(S)$ using a maximization problem of a polyhedral convex function over a convex set. Noting that several papers have been devoted to this topic, we refer to $[2,3,15,16,17,19,20]$. Finally, we give an algorithm for this case. The paper consists of five sections.

In Section 2, by introducing a reverse convex program $(\hat{S})$ which is equivalent to $(S)$ under appropriate assumptions, we first establish some fundamental results. Then, we recall other results about reverse convex programs that will be used in the sequel, especially those dealing with stability. Section 3 is devoted to stability results for the problem $(\widehat{S})$. Section 4 , contains the main result, that is, the necessary and sufficient condition for global optimality. In Section 5, we consider the case where the function $f$ is polyhedral and $g$ is linear, and we give an algorithm for this case.

\section{Basic results}

In this section, we establish some basic results, and recall others dealing with stability of reverse convex programs. For $x \in \mathscr{G}$, set

$$
v(x)=\inf _{\substack{y \in \mathbb{R}^{m} \\ g(x, y) \leq 0}} f(x, y),
$$


(which is a convex function since $f$ and $g$ are convex) and we introduce the following reverse convex program:

$(\widehat{S})$

$$
\min _{\substack{(x, t) \in \mathbb{R}^{n} \times \mathbb{R} \\ G(x) \leq 0 \\ v(x)-t \geq 0}} F(x, t)
$$

We make the following assumptions.

(2.1) The set $\mathscr{G}$ is bounded.

(2.2) There exists a compact set $\mathscr{L}$ of $\mathbb{R}^{m}$. such that $Y(x) \subset \mathscr{L}$, for any $x \in \mathscr{G}$.

(2.3) $F(x, \cdot)$ is a nonincreasing function on $\mathbb{R}$, for any $x \in \mathbb{R}^{n}$.

(2.4) The Slater condition: for any $x \in \mathscr{G}$, there exists $y \in \mathbb{R}^{m}$, such that $g(x, y)<0$.

Remark 2.1. The continuity of the functions $f$ and $g$ and assumption (2.2) imply that the marginal function $v$ is well defined and continuous on $\mathscr{G}$ (see, e.g., [8]). Consequently, throughout the paper we always assume that assumption (2.2) is satisfied.

For the sake of simplicity, we will use the term "solution" instead of "global solution." First, we begin by the following proposition that establishes the existence of solutions to $(\widehat{S})$.

Proposition 2.2. Let assumptions (2.1) and (2.2) hold. Then, the problem $(\hat{S})$ has at least one solution.

Proof. Since the function $F(\cdot, v(\cdot))$ is continuous on the compact set $\mathscr{G}$, it follows that $(S)$ has at least one solution $x$. Hence $(x, v(x))$ is a solution of $(\widehat{S})$.

We have the following equivalence between the problems $(S)$ and $(\hat{S})$.

Proposition 2.3. Let assumptions (2.1), (2.2), and (2.3) hold. Then, the following properties hold:

(i) if $(\hat{x}, \hat{t})$ solves $(\hat{S})$, then $\hat{x}$ solves $(S)$,

(ii) if $\hat{x}$ solves $(S)$, then $(\hat{x}, v(\hat{x}))$ solves $(\hat{S})$.

Proof. (i) Assume that $(\hat{x}, \hat{t})$ solves $(\hat{S})$. Then, $G(\hat{x}) \leq 0$. Let $x \in \mathbb{R}^{n}$ such that $G(x) \leq 0$. So $(x, v(x))$ is a feasible point of $(\hat{S})$, and $F(\hat{x}, \hat{t}) \leq F(x, v(x))$. On the other hand, we have $F(\hat{x}, v(\hat{x})) \leq F(\hat{x}, \hat{t}) \leq F(x, v(x))$, and hence $\hat{x}$ solves $(S)$.

(ii) Assume that $\hat{x}$ solves $(S)$. Then, it is easy to see that $(\hat{x}, v(\hat{x}))$ is feasible for $(\hat{S})$. Let $(x, t)$ be a feasible point of $(\hat{S})$. Then, $G(x) \leq 0$, and $v(x) \geq t$. Since $\hat{x}$ is a solution of $(S)$, we have $F(\hat{x}, v(\hat{x})) \leq F(x, v(x)) \leq F(x, t)$, and therefore $(\hat{x}, v(\hat{x}))$ is a solution of $(\hat{S})$.

Set

$$
Y_{j}=\left\{(x, y) \in \mathbb{R}^{n} \times \mathbb{R}^{m} / g_{j}(x, y) \leq 0\right\}, \quad Y=\bigcap_{j=1}^{q} Y_{j} .
$$


Let $N_{Y_{j}}(x, y)$ be the usual normal cone of $Y_{j}$ at $(x, y)$, let int $Y_{j}$ the interior of the set $Y_{j}$, and let $\delta_{Y_{j}}$ be the indicator function of $Y_{j}$ :

$$
\delta_{Y_{j}}(x, y)= \begin{cases}0 & \text { if } g_{j}(x, y) \leq 0 \\ +\infty & \text { otherwise }\end{cases}
$$

We also have the following result.

Lemma 2.4. Let assumptions (2.2) and (2.4) hold. Then, for any $x \in \mathscr{G}$, there exists $y$ verifying $g(x, y) \leq 0$, and $v(x)=f(x, y)$, such that

$$
\partial v(x) \subset \partial_{x} f(x, y)+\sum_{j=1}^{q} \bigcup_{\lambda_{j} \geq 0} \lambda_{j} \partial_{x} g_{j}(x, y),
$$

where "J" stands for the subdifferential.

Proof. From [1], we have

$$
u^{*} \in \partial v(x) \Longleftrightarrow\left(u^{*}, 0\right) \in \partial\left(f+\sum_{j=1}^{q} \delta_{Y_{j}}\right)(x, y),
$$

where $y$ satisfies $g(x, y) \leq 0$, and $v(x)=f(x, y)$. Then, by using the continuity of finite convex functions on $\mathbb{R}^{n}$, together with the Slater condition (2.4), we get

$$
\bigcap_{j=1}^{q} \operatorname{int} Y_{j} \neq \varnothing
$$

Since $\operatorname{dom} f=\mathbb{R}^{n} \times \mathbb{R}^{m}$, and $\operatorname{dom} \delta_{Y_{j}}=Y_{j}$, then

$$
\partial\left(f+\sum_{j=1}^{q} \delta_{Y_{j}}\right)(x, y)=\partial f(x, y)+\sum_{j=1}^{q} \partial \delta_{Y_{j}}(x, y)=\partial f(x, y)+\sum_{j=1}^{q} N_{Y_{j}}(x, y) .
$$

By [9, Corollary 23.7.1], we have

$$
N_{Y_{j}}(x, y)= \begin{cases}\bigcup \lambda_{j} \partial g_{j}(x, y) & \text { if } g_{j}(x, y)=0 \\ \lambda_{j} \geq 0 & \text { if } g_{j}(x, y)<0\end{cases}
$$

Hence

$$
\begin{aligned}
\partial f(x, y)+\sum_{j=1}^{q} N_{Y_{j}}(x, y) & \subset \partial f(x, y)+\sum_{j=1}^{q} \bigcup_{\lambda_{j} \geq 0} \lambda_{j} \partial g_{j}(x, y) \\
& \subset \partial_{x} f(x, y) \times \partial_{y} f(x, y)+\sum_{j=1}^{q} \bigcup_{\lambda_{j} \geq 0} \lambda_{j}\left[\partial_{x} g_{j}(x, y) \times \partial_{y} g_{j}(x, y)\right] .
\end{aligned}
$$


Finally, property (2.6) implies that

$$
\partial v(x) \subset \partial_{x} f(x, y)+\sum_{j=1}^{q} \bigcup_{\lambda_{j} \geq 0} \lambda_{j} \partial_{x} g_{j}(x, y) .
$$

We recall the following definitions and results from $[4,18]$ that will be used in the sequel.

Let $\hat{f}, \hat{g}, \hat{G}: \mathbb{R}^{n} \rightarrow \mathbb{R}, \alpha, \beta \in \mathbb{R}$, and consider the following problems which are in duality in the following sense:

$\left(\mathscr{P}_{\beta}\right)$

$$
\begin{aligned}
& \min _{\substack{x \in \mathbb{R}^{n} \\
\hat{G}(x) \leq 0 \\
\hat{g}(x) \geq \beta}} \hat{f}(x), \\
& \text { (n) }
\end{aligned}
$$

$\left(2_{\alpha}\right)$

$$
\max _{\substack{x \in \mathbb{R}^{n} \\ \hat{G}(x) \leq 0 \\ \hat{f}(x) \leq \alpha}} \hat{g}(x) .
$$

Let inf $\mathscr{P}_{\beta}$ and sup $\mathscr{Q}_{\alpha}$ denote the optimal values of $\left(\mathscr{P}_{\beta}\right)$ and $\left(\mathscr{Q}_{\alpha}\right)$, respectively, and set

$$
\widehat{\mathscr{D}}=\left\{x \in \mathbb{R}^{n} / \hat{G}(x) \leq 0\right\} .
$$

Definition 2.5. (a) The problem $\left(\mathscr{P}_{\beta}\right)$ is stable if $\lim _{\beta^{\prime} \rightarrow \beta^{+}} \inf \mathscr{P}_{\beta^{\prime}}=\inf \mathscr{P}_{\beta}$.

(b) The problem $\left(2_{\alpha}\right)$ is stable if $\lim _{\alpha^{\prime} \rightarrow \alpha^{-}} \sup 2_{\alpha^{\prime}}=\sup 2_{\alpha}$.

Definition 2.6. A feasible point $x$ of $\left(\mathscr{P}_{\beta}\right)$ is said to be regular for $\left(\mathscr{P}_{\beta}\right)$, if there exists a sequence $\left(x_{k}\right)$ converging to $x$ such that $x_{k} \in \hat{D}$, and $\hat{g}\left(x_{k}\right)>\beta$ for large $k$. Similarly, a feasible point $x$ of $\left(2_{\alpha}\right)$, is said to be regular for $\left(2_{\alpha}\right)$ if there exists a sequence $\left(x_{k}\right)$ converging to $x$ such that $x_{k} \in \hat{D}$, and $\hat{f}\left(x_{k}\right)<\alpha$ for large $k$.

Proposition 2.7. If $\hat{f}$ is upper semicontinuous and if there exists at least one solution of $\left(\mathscr{P}_{\beta}\right)$ that is regular for $\left(\mathscr{P}_{\beta}\right)$, then $\left(\mathscr{P}_{\beta}\right)$ is stable. Similarly, if $\hat{g}$ is lower semicontinuous and if there exists at least one solution of $\left(2_{\alpha}\right)$ that is regular for $\left(2_{\alpha}\right)$, then $\left(2_{\alpha}\right)$ is stable.

Proposition 2.8. (i) Assume that $\left(\mathscr{Q}_{\alpha}\right)$ is stable. Then, $\alpha \leq \inf \mathscr{P}_{\beta} \Rightarrow \beta \geq \sup \mathscr{Q}_{\alpha}$.

(ii) Assume that $\left(\mathscr{P}_{\beta}\right)$ is stable. Then, $\beta \geq \sup \mathscr{2}_{\alpha} \Rightarrow \alpha \leq \inf \mathscr{P}_{\beta}$.

\section{Stability results}

This section is devoted to some stability results for problem $(\hat{S})$ that we will use in the next section. 
Let $G_{i}^{\prime}(x ; d)$ denote the directional derivative of $G_{i}$ at $x$ in the direction $d \in \mathbb{R}^{n}$, and let $I(x)$ denote the index set of active constraints $G_{i}$ at $x, i=1, \ldots, p$, that is, $I(x)=\{i \in$ $\left.\{1, \ldots, p\} / G_{i}(x)=0\right\}$. For $k \in\{1, \ldots, p\}$, set

$$
\begin{gathered}
\mathscr{D}_{k}=\bigcup_{\substack{1, \ldots, k \\
\max }}\left\{x \in \operatorname{Gup}_{\substack{(x, y) \in \mathbb{R}^{n} \times \mathbb{R}^{m} \\
G(x) \leq 0 \\
g(x, y) \leq 0}} f(x, y), \quad f_{\min }=\inf _{\substack{(x, y) \in \mathbb{R}^{n} \times \mathbb{R}^{m} \\
G(x) \leq 0 \\
g(x, y) \leq 0}} f(x, y) .\right.
\end{gathered}
$$

We make the following assumption:

(A1)

$$
\exists l \in\{1, \ldots, p\}, \quad \exists f_{l} \geq f_{\max }, \quad \exists t(l) \leq f_{\min }, \quad \exists x(l) \in \mathscr{G}
$$

such that

(1) $F(x(l), t(l))<\inf _{(x, t) \in \mathscr{D}_{l} \times \mathbb{R}} F(x, t)$, and for any $(x, y) \in \mathscr{G}_{l} \times \mathbb{R}^{m}$, such that $g(x, y) \leq 0$, we have

(2) $0 \notin \bigcup_{i \in I(x)} \partial G_{i}(x)$,

(3) $\partial_{x} f(x, y) \subset \bigcap_{i \in I(x)}\left\{d \in \mathbb{R}^{n} / G_{i}^{\prime}(x ; d)<0\right\}, \bigcup_{j=1, \ldots, q} \partial_{x} g_{j}(x, y) \subset \bigcap_{i \in I(x)}\{d \in$ $\left.\mathbb{R}^{n} / G_{i}^{\prime}(x ; d) \leq 0\right\}$.

Remark 3.1. (1) From the definition of $\varphi_{l}$, we have $\bigcup_{x \in \mathscr{G}_{l}} I(x) \subset\{l+1, \ldots, p\}$.

(2) Assumption (A1) implies that $(x(l), t(l))$ is a feasible point of $(\widehat{S})$, and

$$
F(x(l), t(l))<\inf _{\substack{(x, t) \in \mathscr{D}, \times \mathbb{R} \\(x) \geq t}} F(x, t) .
$$

Furthermore, if $(\hat{x}, \hat{t})$ solves $(\hat{S})$, then $(\hat{x}, \hat{t}) \notin\left\{(x, t) \in \mathscr{D}_{l} \times \mathbb{R} / v(x) \geq t\right\}$.

(3) The set $\left\{d \in \mathbb{R}^{n} / G_{i}^{\prime}(x ; d)<0\right\}$ of descent directions of active constraints $G_{i}$ at $x$ is nonempty for any $i \in I(x)$, if and only if the condition (2) of assumption (A1) is satisfied. On the other hand, we have

$$
\begin{aligned}
\left\{d \in \mathbb{R}^{n} / G_{i}^{\prime}(x ; d) \leq 0\right\} & =\left\{d \in \mathbb{R}^{n} /\left\langle x^{*}, d\right\rangle \leq 0, \forall x^{*} \in \partial G_{i}(x)\right\} \\
& =\left\{d \in \mathbb{R}^{n} /\left\langle\lambda x^{*}, d\right\rangle \leq 0, \forall \lambda \geq 0, \forall x^{*} \in \partial G_{i}(x)\right\} \\
& =\left[\mathbb{R}^{+} \partial G_{i}(x)\right]^{\circ},
\end{aligned}
$$

where $\left[\mathbb{R}^{+} \partial G_{i}(x)\right]^{\circ}$ denotes the polar cone of $\mathbb{R}^{+} \partial G_{i}(x)$. Then, condition (2) of assumption (A1) implies

$$
\left\{d \in \mathbb{R}^{n} / G_{i}^{\prime}(x ; d)<0\right\}=\operatorname{int}\left[\mathbb{R}^{+} \partial G_{i}(x)\right]^{\circ} .
$$

Remark 3.2. Assume that assumptions of Proposition 2.2 and assumption (A1) hold. Let $(\bar{x}, \bar{t})$ be a solution of $(\hat{S})$. Hence, $\bar{x} \in \varphi_{l}$ and $v(\bar{x}) \geq \bar{t}$. We show that if $I(\bar{x})=\varnothing$, then $(\bar{x}, \bar{t})$ is regular for $(\hat{S})$. Let $\left(x_{k}, t_{k}\right)=(\bar{x}, \bar{t}-1 / k), k \in \mathbb{N}^{*}$, which converges to $(\bar{x}, \bar{t})$, and satisfies $v\left(x_{k}\right)>t_{k}, G\left(x_{k}\right)<0$, for any $k \in \mathbb{N}^{*}$. Therefore, $(\bar{x}, \bar{t})$ is regular. 
In Propositions 3.3-3.6, in order to show that the problem $(\widehat{S})$ is stable, we will first show that any solution $(\bar{x}, \bar{t})$ of $(\widehat{S})$ is regular.

Proposition 3.3. Let assumptions (2.1)-(2.4) and (A1) hold. Then, the problem $(\hat{S})$ is stable.

Proof. According to Remark 3.2, and without loss of generality, we will show the regularity of any solution $(\bar{x}, \bar{t})$ to $(\widehat{S})$ assuming that $I(\bar{x}) \neq \varnothing$. Let $x^{*} \in \partial v(\bar{x})$. From Lemma 2.4, there exist $\left(u^{*}, v_{j}^{*}\right) \in \partial_{x} f(\bar{x}, y) \times \partial_{x} g_{j}(\bar{x}, y)$, and $\lambda_{j} \geq 0, j=1, \ldots, q$, such that

$$
x^{*}=u^{*}+\sum_{j=1}^{q} \lambda_{j} v_{j}^{*},
$$

where $y \in \mathbb{R}^{m}$ satisfies $g(\bar{x}, y) \leq 0$, and $v(\bar{x})=f(\bar{x}, y)$. Let $i \in I(\bar{x})$. We have $\operatorname{dom} G_{i}=\mathbb{R}^{n}$, hence the directional derivative $G_{i}^{\prime}\left(\bar{x} ; x^{*}\right)$ exists and verifies

$$
G_{i}^{\prime}\left(\bar{x} ; x^{*}\right) \leq G_{i}^{\prime}\left(\bar{x} ; u^{*}\right)+\sum_{j=1}^{q} \lambda_{j} G_{i}^{\prime}\left(\bar{x} ; v_{j}^{*}\right)<0,
$$

where the last strict inequality follows from assumption (A1) (since $\bar{x} \in \mathscr{G}_{l}$ ). Then, from (3.7) we deduce that $x^{*}$ is a descent direction of $G_{i}$ at $\bar{x}$, and hence $x^{*} \neq 0$. Let $x_{k}=$ $\bar{x}+\alpha_{k} x^{*}, t_{k}=\bar{t}$, for all $k \in \mathbb{N}$, with $\alpha_{k} \backslash 0^{+}$. Then, $\left(x_{k}, t_{k}\right) \rightarrow(\bar{x}, \bar{t})$, as $k \rightarrow+\infty$. It follows that

$$
G_{i}\left(\bar{x}+\alpha_{k} x^{*}\right)-G_{i}(\bar{x})<0 \quad \text { for large } k
$$

That is,

$$
G_{i}\left(x_{k}\right)<G_{i}(\bar{x})=0 \quad \text { for large } k .
$$

On the other hand, for $i \notin I(\bar{x})$, it is easy to see that $G_{i}\left(x_{k}\right)<0$, for large $k$. Since $x^{*} \in$ $\partial v(\bar{x})$, then

$$
v\left(x_{k}\right) \geq v(\bar{x})+\alpha_{k}\left\|x^{*}\right\|^{2}>t_{k} \quad \forall k,
$$

where $\|\cdot\|$ denotes the Euclidean norm in $\mathbb{R}^{n}$. Hence $(\bar{x}, \bar{t})$ is regular and the result follows from Proposition 2.7.

In the following example the assumptions of Proposition 3.3 are satisfied.

Example 3.4. Let

$$
\begin{gathered}
\mathscr{L}=[0,10], \quad F(x, t)=e^{10\left(x_{1}+x_{2}\right)-t}, \quad G=\left(G_{1}, G_{2}, G_{3}, G_{4}\right), \\
G_{1}(x)=x_{1}+x_{2}-3, \quad G_{2}(x)=\left|x_{1}+x_{2}-1\right|-2\left(x_{1}+x_{2}\right)-\frac{9}{10}, \\
G_{3}(x)=-x_{1}, \quad G_{4}(x)=-x_{2}, \\
f(x, y)=\left\|x^{T}\right\|+3\left(x_{1}+x_{2}\right)+y, \quad g=\left(g_{1}, g_{2}, g_{3}\right), \\
g_{1}(x, y)=\left\|x^{T}\right\|+2\left(x_{1}+x_{2}\right)-y, \quad g_{2}(x, y)=y-10, \quad g_{3}(x, y)=-y,
\end{gathered}
$$


where $x=\left(x_{1}, x_{2}\right) \in \mathbb{R}^{2}, y, t \in \mathbb{R}$, and $e$ is the exponential function. Hence assumptions (2.1)-(2.4) are satisfied. We verify assumption (A1).

Let $\mathscr{D}_{1}=\left\{x \in \mathscr{G} / G_{1}(x)=0\right\}$, and $\mathscr{G}_{1}=\mathscr{G} \backslash \mathscr{D}_{1}$. We have

$$
f_{\min }=\inf _{\substack{(x, y) \in \mathbb{R}^{2} \times \mathbb{R} \\ G(x) \leq 0 \\ g(x, y) \leq 0}} f(x, y) \geq 0, \quad f_{\max }=\max _{\substack{(x, y) \in \mathbb{R}^{2} \times \mathbb{R} \\ G(x) \leq 0 \\ g(x, y) \leq 0}} f(x, y)=22 .
$$

Let $f_{1}=22, t(1)=0 \leq f_{\min }$, and $x(1)=(1 / 2,0)$. Then,

$$
F(x(1), t(1))=e^{5}<\inf _{\substack{(x, t) \in \mathscr{T}_{1} \times \mathbb{R} \\ t \leq 22}}\left\{e^{10\left(x_{1}+x_{2}\right)-t}\right\}=e^{8} .
$$

Let $(x, y) \in \mathscr{G}_{1} \times \mathbb{R}$, such that $g(x, y) \leq 0$. We have $I(x) \subset\{2,3,4\}$, and

$$
\partial G_{2}(x)= \begin{cases}{[-1,1](1,1)^{T}-\left\{(2,2)^{T}\right\}=\left\{-\alpha(1,1)^{T}, 1 \leq \alpha \leq 3\right\}} & \text { if } x_{1}+x_{2}=1 \\ \left\{(-3,-3)^{T}\right\} & \text { if } x_{1}+x_{2}<1 \\ \left\{(-1,-1)^{T}\right\} & \text { if } x_{1}+x_{2}>1\end{cases}
$$

According to Remark 3.1, we have $\left\{d \in \mathbb{R}^{2} / G_{2}^{\prime}(x ; d)<0\right\}=\mathbb{R}_{+}^{*} \times \mathbb{R}_{+}^{*}$, and

$$
\left\{d \in \mathbb{R}^{2} / G_{2}^{\prime}(x ; d) \leq 0\right\}=\mathbb{R}_{+}^{2} .
$$

On the other hand, we have $\nabla G_{3}(x)=(-1,0)^{T}$, and $\nabla G_{4}(x)=(0,-1)^{T}$.

$$
\begin{aligned}
& \partial_{x} f(x, y)= \begin{cases}B(0,1)+\left\{(3,3)^{T}\right\} & \text { if } x=0, \\
\left\{\frac{x^{T}}{\left\|x^{T}\right\|}+(3,3)^{T}\right\} & \text { if } x \neq 0,\end{cases} \\
& \partial_{x} g_{1}(x, y)=\left\{\begin{array}{ll}
B(0,1)+\left\{(2,2)^{T}\right\} & \text { if } x=0, \\
\left\{\frac{x^{T}}{\left\|x^{T}\right\|}+(2,2)^{T}\right\} & \text { if } x \neq 0,
\end{array} \quad \nabla_{x} g_{2}(x, y)=\nabla_{x} g_{3}(x, y)=\left(\begin{array}{l}
0 \\
0
\end{array}\right),\right.
\end{aligned}
$$

where $B(0,1)$ denotes the Euclidean unit ball of $\mathbb{R}^{2}$. Then,

$$
\begin{gathered}
\partial_{x} f(x, y) \subset\left\{d \in \mathbb{R}^{2} / G_{2}^{\prime}(x ; d)<0\right\}, \\
\partial_{x} g_{1}(x, y) \cup\left\{\nabla_{x} g_{2}(x, y), \nabla_{x} g_{3}(x, y)\right\} \subset\left\{d \in \mathbb{R}^{2} / G_{2}^{\prime}(x ; d) \leq 0\right\}, \\
\left\langle\nabla G_{i}(x), u^{*}\right\rangle<0, \quad\left\langle\nabla G_{i}(x), v_{j}^{*}\right\rangle \leq 0,
\end{gathered}
$$

for any $\left(u^{*}, v_{j}^{*}\right) \in \partial_{x} f(x, y) \times \partial_{x} g_{j}(x, y), i=3,4$ and $j=1,2,3$. Furthermore, $0 \notin$ $\partial G_{2}(x) \cup\left\{\nabla G_{3}(x), \nabla G_{4}(x)\right\}$. Then, $0 \notin \bigcup_{i \in I(x)} \partial G_{i}(x)$. Hence, assumption (A1) is satisfied. 
Proposition 3.5. Proposition 3.3 holds if assumption (A1) is replaced by the following qualification condition:

(A2) $\exists l \in\{1, \ldots, p\}, \exists f_{l} \geq f_{\max }, \exists t(l) \leq f_{\min }, \exists x(l) \in \mathscr{G}_{\text {such that }}$

(1) $F(x(l), t(l))<\inf _{(x, t) \in \mathscr{D}_{l} \times \mathbb{R}} F(x, t)$, and for any $(x, y) \in \mathscr{G}_{l} \times \mathbb{R}^{m}$, such that $g(x, y) \leq 0$, and $\left(u, v_{j}\right) \in \partial_{x} f(x, y) \times \partial_{x} g_{j}(x, y), j=1, \ldots, q$, it holds that

(2) $0 \notin \bigcup_{i \in I(x)} \partial G_{i}(x)$,

(3) $\left\langle w_{i}, u\right\rangle \geq 0,\left\langle w_{i}, v_{j}\right\rangle \geq 0$, for any descent direction $w_{i}$ of $G_{i}$ at $x, i \in I(x)$, where $\langle\cdot, \cdot\rangle$ denotes the inner product of two vectors.

Proof. The proof is essentially identical to the one of Proposition 3.3. We only give some arguments concerning the modifications. Let $i \in I(\bar{x}), x^{*} \in \partial v(\bar{x})$, and $w^{*}$ be a descent direction of $G_{i}$ at $\bar{x}$. Define $x_{k}=\bar{x}+\alpha_{k} w^{*}, \alpha_{k} \searrow 0^{+}$, and $t_{k}=\bar{t}-1 / k$, for all $k \geq 1$. Then, $\left(x_{k}, t_{k}\right) \rightarrow(\bar{x}, \bar{t})$, as $k \rightarrow+\infty$. From Lemma 2.4, there exist $u^{*} \in \partial_{x} f(\bar{x}, y), v_{j}^{*} \in \partial_{x} g_{j}(\bar{x}, y)$, and $\lambda_{j} \geq 0$, such that $x^{*}=u^{*}+\sum_{j=1}^{q} \lambda_{j} v_{j}^{*}$, where $y$ satisfies $g(\bar{x}, y) \leq 0$, and $f(\bar{x}, y)=$ $v(\bar{x})$. Since $x^{*} \in \partial v(\bar{x})$, it follows that

$$
v\left(x_{k}\right) \geq v(\bar{x})+\alpha_{k}\left\langle x^{*}, w^{*}\right\rangle=v(\bar{x})+\alpha_{k}\left\langle u^{*}, w^{*}\right\rangle+\alpha_{k} \sum_{j=1}^{q} \lambda_{j}\left\langle v_{j}, w^{*}\right\rangle .
$$

Then, by assumption (A2), we have $v\left(x_{k}\right) \geq v(\bar{x})>t_{k}$, for large $k$. On the other hand, from the definition of $w^{*}$, it follows that $G_{i}\left(x_{k}\right)<G_{i}(\bar{x})=0$, for large $k$. The end of the proof is identical to the end of that of Proposition 3.3.

Proposition 3.6. Proposition 3.3 holds if assumption (A1) is replaced by the following qualification condition:

(A3) $\exists l \in\{1, \ldots, p\}, \exists f_{l} \geq f_{\max }, \exists t(l) \leq f_{\min }, \exists x(l) \in G_{\text {such that }}$

(1) $F(x(l), t(l))<\inf _{(x, t) \in \mathscr{D}_{l} \times \mathbb{R}, \leq f_{l}} F(x, t)$, and for any $(x, y) \in \mathscr{G}_{l} \times \mathbb{R}^{m}$, such that $g(x, y) \leq 0$

(2) the functions $f$ and $g$ are differentiable at $(x, y)$, and for any $i \in I(x)$, the function $G_{i}$ is differentiable at $x$, and satisfies

(i) $\left\langle\nabla G_{i}(x), \nabla_{x} f(x, y)\right\rangle\left\langle 0,\left\langle\nabla G_{i}(x), \nabla_{x} g_{j}(x, y)\right\rangle \leq 0\right.$, for all $j$, where $\nabla$ stands for the gradient,

(ii) $0 \notin\left\{\nabla G_{i}(x), i \in I(x)\right\}$.

Proof. In the differentiable case and according to [14, Theorem 2.1], the set $\partial v(\bar{x})$ becomes

$$
\begin{gathered}
\partial v(\bar{x})=\left\{\nabla_{x} f(\bar{x}, y)+\sum_{j=1}^{q} \lambda_{j} \nabla_{x} g_{j}(\bar{x}, y), \text { such that } \nabla_{y} f(\bar{x}, y)+\sum_{j=1}^{q} \lambda_{j} \nabla_{y} g_{j}(\bar{x}, y)=0,\right. \\
\left.g(\bar{x}, y) \leq 0, v(\bar{x})=f(\bar{x}, y), \lambda_{j} \geq 0, \text { with } \lambda_{j}=0, \text { if } g_{j}(\bar{x}, y)<0\right\} .
\end{gathered}
$$


Therefore, for $x^{*} \in \partial v(\bar{x})$, there exists $\lambda_{j} \geq 0, j=1, \ldots, q$, with $\lambda_{j}=0$, if $g_{j}(\bar{x}, y)<0$, such that

$$
x^{*}=\nabla_{x} f(\bar{x}, y)+\sum_{j=1}^{q} \lambda_{j} \nabla_{x} g_{j}(\bar{x}, y) .
$$

Let $i \in I(\bar{x})$. Using assumption (A3), we get

$$
\left\langle\nabla G_{i}(\bar{x}), x^{*}\right\rangle<0
$$

So, $x^{*}$ is a descent direction of $G_{i}$ at $\bar{x}$, and hence $x^{*} \neq 0$. Let $\left(x_{k}, t_{k}\right) \rightarrow(\bar{x}, \bar{t})$, as $k \rightarrow+\infty$, be the sequence defined in Proposition 3.3, $x_{k}=\bar{x}+\alpha_{k} x^{*}, \alpha_{k} \searrow 0^{+}$, that satisfies $v\left(x_{k}\right)>t_{k}$, for large $k$. Since the function $G_{i}$ is differentiable at $\bar{x}$, it follows that

$$
G_{i}\left(x_{k}\right)=G_{i}(\bar{x})+\alpha_{k}\left\langle\nabla G_{i}(\bar{x}), x^{*}\right\rangle+\alpha_{k}\left\|x^{*}\right\| \beta\left(\bar{x}, \alpha_{k} x^{*}\right),
$$

where $\beta\left(\bar{x}, \alpha_{k} x^{*}\right) \rightarrow 0$, as $k \rightarrow+\infty$. Combining (3.21) and (3.22) yields $G_{i}\left(x_{k}\right)<G_{i}(\bar{x})=$ 0 for large $k$. On the other hand, for $i \notin I(\bar{x})$, we have $G_{i}\left(x_{k}\right)<0$, for large $k$. Hence, $(\bar{x}, \bar{t})$ is regular and the result follows from Proposition 2.7.

We give the following example where assumptions of Proposition 3.6 are satisfied.

Example 3.7. Let

$$
\begin{gathered}
\mathscr{L}=[0,4], \quad F(x, t)=e^{3\left(x_{1}+x_{2}\right)-t,} \quad G=\left(G_{1}, G_{2}, G_{3}, G_{4}\right), \\
G_{1}(x)=x_{1}+x_{2}-3, \quad G_{2}(x)=x_{1}-2 x_{2}-2, \quad G_{3}(x)=-x_{1}, \quad G_{4}(x)=-x_{2}, \\
f(x, y)=x_{1}+x_{2}+y, \quad g=\left(g_{1}, g_{2}, g_{3}\right), \\
g_{1}(x, y)=x_{1}+x_{2}-y, \quad g_{2}(x, y)=y-4, \quad g_{3}(x, y)=-y,
\end{gathered}
$$

where $x=\left(x_{1}, x_{2}\right) \in \mathbb{R}^{2}, y, t \in \mathbb{R}$. We easily verify that assumptions (2.1)-(2.4) are satisfied. We verify assumption (A3).

Let $\mathscr{D}_{1}=\left\{x \in \mathscr{G} / G_{1}(x)=0\right\}$ and $\mathscr{G}_{1}=\mathscr{G} \backslash \mathscr{D}_{1}$. We have

$$
f_{\min }=\inf _{\substack{(x, y) \in \mathbb{R}^{2} \times \mathbb{R} \\ G(x) \leq 0 \\ g(x, y) \leq 0}} f(x, y)=0, \quad f_{\max }=\max _{\substack{(x, y) \in \mathbb{R}^{2} \times \mathbb{R} \\ G(x) \leq 0 \\ g(x, y) \leq 0}} f(x, y)=7 .
$$

Let $f_{1}=7, t(1)=0 \leq f_{\min }$, and $x(1)=(0,0)$. Then,

$$
F(x(1), t(1))=1<\inf _{\substack{(x, t) \in \mathscr{D}_{1} \times \mathbb{R} \\ t \leq 7}}\left\{e^{3\left(x_{1}+x_{2}\right)-t}\right\}=e^{2} .
$$

Let $(x, y) \in \mathscr{G}_{1} \times \mathbb{R}$, such that $g(x, y) \leq 0$. We have $I(x) \subset\{2,3,4\}$, and

$$
\begin{gathered}
\nabla G_{2}(x)=(1,-2)^{T}, \quad \nabla G_{3}(x)=(-1,0)^{T}, \quad \nabla G_{4}(x)=(0,-1)^{T}, \\
\nabla_{x} f(x, y)=\nabla g_{1}(x, y)=(1,1)^{T}, \quad \nabla_{x} g_{2}(x, y)=\nabla_{x} g_{3}(x, y)=(0,0)^{T} .
\end{gathered}
$$


Then,

$$
\left\langle\nabla G_{i}(x), \nabla_{x} f(x, y)\right\rangle<0, \quad\left\langle\nabla G_{i}(x), \nabla_{x} g_{j}(x, y)\right\rangle \leq 0, \quad \forall i \in\{2,3,4\}, \forall j \in\{1,2,3\},
$$

and $0 \notin\left\{\nabla G_{i}(x), i \in I(x)\right\}$. Hence, assumption (A3) is satisfied.

\section{Global optimality condition}

In this section, we provide a necessary and sufficient condition for global optimality.

This result is essentially based on the stability results given for $(\hat{S})$ in the previous section. Set

$$
\mathscr{E}=\left\{(x, t) \in \mathscr{G} \times \mathbb{R} / \exists y \in \mathbb{R}^{m}, \text { such that } g(x, y) \leq 0, f(x, y) \geq t\right\}
$$

and make the following assumptions:

(4.1) for any $x \in \mathscr{G}, \lim _{t \rightarrow-\infty} F(x, t)=+\infty$,

(4.2) there exists $(\bar{x}, \bar{t}, \bar{y}) \in \mathscr{G} \times \mathbb{R} \times \mathbb{R}^{m}$, such that

(i) $f(\bar{x}, \bar{y})<\bar{t}, g(\bar{x}, \bar{y}) \leq 0$,

(ii) $F(\bar{x}, \bar{t})<\inf _{(x, t) \in \mathscr{E}} F(x, t)$.

Remark 4.1. Assumption (4.2) implies that there exists $(\bar{x}, \bar{t}) \in \mathscr{G} \times \mathbb{R}$ such that $G(\bar{x}) \leq 0$, $v(\bar{x})<\bar{t}$, and

$$
F(\bar{x}, \bar{t})<\inf _{\substack{(x, t) \in \mathbb{R}^{n} \times \mathbb{R} \\ G(x) \leq 0 \\ v(x) \geq t}} F(x, t) .
$$

Theorem 4.2. Assume that assumptions (2.1)-(2.3), (4.1) and (4.2) hold. Let $\hat{x}$ be a feasible point of $(S)$.

(i) If $\hat{x}$ solves $(S)$, then it is necessary that

$(\mathrm{OC})$

$$
\max _{\substack{(x, t) \in \mathbb{R}^{n} \times \mathbb{R} \\ G(x) \leq 0 \\ F(x, t) \leq F(\hat{x}, v(\hat{x}))}} \min _{\substack{y \in \mathbb{R}^{m} \\ g(x, y) \leq 0}}[f(x, y)-t]=0 .
$$

(ii) If moreover, assumption (2.4) and one of assumptions (A1)-(A3) are satisfied, then the condition $(\mathrm{OC})$ becomes sufficient.

Proof. (i) From Proposition 2.3, we have $\hat{x}$ solution of $(S)$ if and only if $(\hat{x}, v(\hat{x}))$ is a solution of $(\hat{S})$. Let $\left(\mathscr{P}_{\beta}\right), \beta=0$, and $\left(\mathscr{Q}_{\alpha}\right), \alpha=F(\hat{x}, v(\hat{x}))$, denote, respectively, the problem $(\widehat{S})$ and the problem

$$
\max _{\substack{(x, t) \in \mathbb{R}^{n} \times \mathbb{R} \\ G(x) \leq 0 \\ F(x, t) \leq F(\hat{x}, v(\hat{x}))}}[v(x)-t] .
$$


Since problem $\left(\mathscr{Q}_{\alpha}\right)$ is stable (see the appendix), and $\alpha=F(\hat{x}, v(\hat{x}))=\inf \hat{S}$, it follows from Proposition 2.8 that

$$
\sup 2_{\alpha}=\max _{\substack{(x, t) \in \mathbb{R}^{n} \times \mathbb{R} \\ G(x) \leq 0 \\ F(x, t) \leq F(\hat{x}, v(\hat{x}))}}[v(x)-t]=\max _{\substack{(x, t) \in \mathbb{R}^{n} \times \mathbb{R} \\ G(x) \leq 0 \\ F(x, t) \leq F(\hat{x}, v(\hat{x}))}} \min _{\substack{y \in \mathbb{R}^{m} \\ g(x, y) \leq 0}}[f(x, y)-t] \leq 0 .
$$

Also, since $(\hat{x}, v(\hat{x}))$ is feasible for $\left(\mathscr{Q}_{\alpha}\right)$, it follows that $\sup \mathscr{2}_{\alpha} \geq 0$, and hence sup $\mathscr{2}_{\alpha}=0$.

(ii) Under the additional assumptions (2.4) and one of assumptions (A1)-(A3), the problem $(\hat{S})$ is stable (see Section 3). Since sup $\mathscr{2}_{\alpha}=0$, it follows that $\alpha=F(\hat{x}, v(\hat{x})) \leq$ $\inf \hat{S}$. Hence $(\hat{x}, v(\hat{x}))$ solves $(\hat{S})$, and $\hat{x}$ solves $(S)$.

Consequently, Theorem 4.2 opens another way for the use of a possible numerical resolution of $(S)$, if of course the problem

$$
\max _{\substack{(x, t) \in \mathbb{R}^{n} \times \mathbb{R} \\ G(x) \leq 0 \\ F(x, t) \leq F(\hat{x}, v(\hat{x}))}} \min _{\substack{y \in \mathbb{R}^{m} \\ g(x, y) \leq 0}}[f(x, y)-t]
$$

belongs to a class for which a method of resolution of min-max problems can be applied. As mentioned in the introduction, for papers dealing with max-min solutions in the linked constraints case, we refer to $[5,7,10,11]$.

\section{The polyhedral case}

Let $f$ and $g$ be the functions defined by

$$
\begin{gathered}
f(x, y)=\max _{i=1, \ldots, r}\left[\left\langle A_{i}, x\right\rangle+\left\langle B_{i}, y\right\rangle-c_{i}\right], \\
g(x, y)=C x+D y-e
\end{gathered}
$$

where

$$
\begin{gathered}
A_{i}=\left(a_{i 1}, \ldots, a_{i n}\right)^{T}, \quad B_{i}=\left(b_{i 1}, \ldots, b_{i m}\right)^{T}, \quad c_{i} \in \mathbb{R}, i=1, \ldots, r, \\
C \in \mathbb{R}^{q \times n}, \quad D \in \mathbb{R}^{q \times m}, \quad e \in \mathbb{R}^{q},
\end{gathered}
$$

and $T$ stands for the transposition. Let $A$ and $B$ denote the matrices with the $i$ th rows being equal to $A_{i}^{T}$ and $\left(B_{i}^{T},-1\right)$, respectively, $\left(A \in \mathbb{R}^{r \times n}\right.$ and $\left.B \in \mathbb{R}^{r \times(m+1)}\right)$. Set

$$
\begin{gathered}
z=\left(y^{T}, t\right)^{T}, \quad \hat{c}=(0, \ldots, 0,1)^{T} \in \mathbb{R}^{m+1}, \quad c=\left(c_{1}, \ldots, c_{r}\right)^{T}, \\
\hat{A}=\left(\begin{array}{l}
A \\
C
\end{array}\right) \in \mathbb{R}^{(r+q) \times n}, \quad \hat{B}=\left(\begin{array}{c}
B \\
(D, 0)
\end{array}\right) \in \mathbb{R}^{(r+q) \times(m+1)}, \quad d=\left(\begin{array}{l}
c \\
e
\end{array}\right) \in \mathbb{R}^{r+q} .
\end{gathered}
$$

Then,

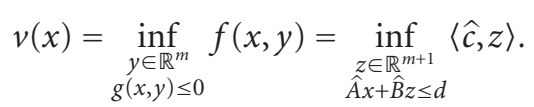

The following result shows that $v$ is a polyhedral convex function. 
Proposition 5.1. Under assumption (2.2), the marginal function $v$ has the following expression:

$$
v(x)=\max _{j=1, \ldots, s}\left[\left\langle\hat{A}^{T} u_{j}, x\right\rangle-\left\langle d, u_{j}\right\rangle\right]
$$

where $u_{1}, \ldots, u_{s}$ are the vertices of the polyhedral set $\left\{u \in \mathbb{R}_{+}^{r+q} \backslash\{0\} / \hat{B}^{T} u=-\hat{c}\right\}$.

Proof. By the duality theorem of linear programming, we have

$$
v(x)=\max _{\substack{u \in \mathbb{R}^{r+q} \\ \hat{B}^{T} u=-\widehat{c} \\ u \geq 0}}\langle\hat{A} x-d, u\rangle
$$

Let $u_{1}, \ldots, u_{s}$ be the vertices of the polyhedral set $\left\{u \in \mathbb{R}_{+}^{r+q} \backslash\{0\} / \hat{B}^{T} u=-\hat{c}\right\}$. Then

$$
v(x)=\max _{\substack{u \in \mathbb{R}^{r+q} \\ \widehat{B}^{T} u=-\hat{c} \\ u \geq 0}}\langle\hat{A} x-d, u\rangle=\max _{j=1, \ldots, s}\left[\left\langle\widehat{A}^{T} u_{j}, x\right\rangle-\left\langle d, u_{j}\right\rangle\right] .
$$

Then, we give the optimality condition corresponding to this case.

Theorem 5.2. Assume that assumptions (2.1)-(2.3), (4.1) and (4.2) hold. Let $\hat{x}$ be a feasible point of $(S)$.

(i) If $\hat{x}$ solves $(S)$, then it is necessary that $(\widehat{O C})$

$$
\max _{\substack{(x, t) \in \mathbb{R}^{n} \times \mathbb{R} \\ G(x) \leq 0 \\ F(x, t) \leq F(\hat{x}, v(\hat{x}))}}\left[\max _{j=1, \ldots, s}\left[\left\langle\hat{A}^{T} u_{j}, x\right\rangle-\left\langle d, u_{j}\right\rangle\right]-t\right]=0 .
$$

(ii) If moreover, assumption (2.4) and one of assumptions (A1)-(A3) are satisfied, then condition $(\widehat{O C})$ becomes sufficient.

Proof. Apply Theorem 4.2.

Hence, Theorem 5.2 offers the possibility of a resolution of $(S)$ via a maximization problem of a polyhedral convex function over a convex set. As is well known, there are several works related to this topic, we cite, for example, $[2,3,15,16,17,19,20]$ and the book [4].

We give the following algorithm in the case where $F(x, \cdot)$ is a decreasing function for any $x \in \mathscr{G}$, which is an adaptation of the one proposed by Tuy in [18]. We suppose, of course, that assumptions (2.1)-(2.4), one of assumptions (A1)-(A3), and assumptions (4.1) and (4.2) are satisfied.

Let $(\widetilde{w}, \tilde{\alpha})$ be a point verifying the inequality given in Remark 4.1 (see the procedure given in Remark 5.4 below, for finding such a point). 


\section{Algorithm 5.3.}

\section{Initialization}

(I) Start from any point $\left(x_{1}, t_{1}\right)$ such that $G\left(x_{1}\right) \leq 0$, and $v\left(x_{1}\right)=t_{1}$ (we can choose any point $\left(x_{1}, v\left(x_{1}\right)\right)$ satisfying $\left.G\left(x_{1}\right) \leq 0\right)$.

Iteration $k=1,2, \ldots$

(I) Solve the subproblem

$$
\left(Q\left(x_{k}\right)\right)
$$

$$
\max \left\{v(x)-t: G(x) \leq 0, F(x, t) \leq F\left(x_{k}, t_{k}\right)\right\},
$$

and obtain a solution $\left(z_{k}, r_{k}\right)$ to 5.9 .

\section{Test of optimality}

(I) If $v\left(z_{k}\right)=r_{k}$, stop. Then, $z_{k}$ solves $(S)$.

(II) Otherwise, set $\left(x_{k+1}, t_{k+1}\right):=\pi\left(z_{k}, r_{k}\right)$, where

$$
\pi\left(z_{k}, r_{k}\right):=\lambda\left(z_{k}, r_{k}\right)+(1-\lambda)(\tilde{w}, \tilde{\alpha}) \quad \text { with } \lambda \in[0,1],
$$

such that

$$
v\left(\lambda z_{k}+(1-\lambda) \tilde{w}\right)=\lambda r_{k}+(1-\lambda) \tilde{\alpha},
$$

and go to iteration $k+1$.

Remark 5.4. (1) We suggest the following procedure for finding the point $(\tilde{w}, \tilde{\alpha})$ used in Algorithm 5.3. For a fixed $\epsilon>0$, consider the following problem:

$\left(P_{\epsilon}\right)$

$$
\min _{\substack{(x, t) \in \mathbb{R}^{n} \times \mathbb{R} \\ G(x) \leq 0}} F(x, t),
$$

(where $f_{\min }$ and $f_{\max }$ are defined in Section 3 ) and let $(\tilde{w}, \tilde{\alpha})$ be a solution of $\left(P_{\epsilon}\right)$. Using that $F(x, \cdot)$ is a decreasing function, we can easily see that $\tilde{\alpha}=f_{\max }+\epsilon$. If $(\hat{x}, v(\hat{x}))$ is optimal for $(\hat{S})$, then $v(\hat{x}) \leq f_{\max } \leq \tilde{\alpha}$, and $F(\hat{x}, \tilde{\alpha}) \leq F(\hat{x}, v(\hat{x}))$.

On the other hand, $(\hat{x}, \tilde{\alpha})$ is feasible for $(5.12)$, then $F(\tilde{w}, \tilde{\alpha}) \leq F(\hat{x}, \tilde{\alpha})$ and so $F(\tilde{w}, \tilde{\alpha})<$ $F(\hat{x}, v(\hat{x}))$.

(2) The existence of solutions to the problem 5.9 can be justified in the same way as in Step 1 of the proof given in the appendix.

(3) The extremal points of the polyhedral set $\left\{u \in \mathbb{R}_{+}^{r+q} \backslash\{0\} / \widehat{B}^{T} u=-\hat{c}\right\}$, in the formula of the marginal function $v$, can be identified by the simplex method.

\section{Conclusion}

As is well known, some numerical methods already exist in two-level optimization, and can only be applied to some classes of bilevel programming problems. According to our 
study, the sufficient and necessary optimality condition $\left(\mathrm{O}^{\complement} \mathrm{C}\right)$ given for a class of bilevel programs with extremal value function, can open a way for the use of a possible resolution via min-max problems. Besides, as we have seen, when $f$ is polyhedral and $g$ is linear, another possibility appears, it is the one of a maximization problem of a polyhedral convex function over a convex set. In conclusion, for a given problem of the type $(S)$, we first must classify it, and see if it can be solved by the methods of bilevel optimization, or min-max problems with linked constraints, or possibly none of them.

\section{Appendix}

Proof of the stability of $\left(2_{\alpha}\right)$ in Theorem 4.2. The proof is given in two steps. In the first step, we show that problem $\left(2_{\alpha}\right)$ admits solutions, and in the second, we show that any solution is regular, and the result will follow from Proposition 2.7.

Step 1. Since $(\hat{x}, v(\hat{x}))$ solves $(\hat{S})$, we have $G(\hat{x}) \leq 0$, and then $(\hat{x}, v(\hat{x}))$ is a feasible point for the problem

$\left(2_{\alpha}\right)$

$$
\max _{\substack{(x, t) \in \mathbb{R}^{n} \times \mathbb{R} \\ G(x) \leq 0 \\ F(x, t) \leq F(\hat{x}, v)(\hat{x})}}[v(x)-t] .
$$

Hence, $\sup \mathscr{2}_{\alpha} \geq 0$, and we deduce that $\left(2_{\alpha}\right)$ is equivalent to the following problem:

$$
\max _{\substack{(x, t) \in \mathbb{R}^{n} \times \mathbb{R} \\ G(x) \leq 0 \\ F(x, t) \leq F(\hat{x}, v(\hat{x})) \\ v(x) \geq t}}[v(x)-t] .
$$

Set

$$
E_{\alpha}=\left\{(x, t) \in \mathbb{R}^{n} \times \mathbb{R} / G(x) \leq 0, F(x, t) \leq F(\hat{x}, v(\hat{x})), v(x) \geq t\right\} .
$$

Since the function $v(x)-t$ is continuous on $\mathscr{G} \times \mathbb{R}$ (see Remark 2.1), it suffices to show that the set $E_{\alpha}$ is compact. First, we remark that $E_{\alpha}$ is closed. Suppose that $E_{\alpha}$ is not bounded. Then, there exists $\left(x_{n}, t_{n}\right) \in E_{\alpha}$ such that $\left\|\left(x_{n}, t_{n}\right)\right\| \rightarrow+\infty$, as $n \rightarrow+\infty$. Since $x_{n} \in \mathscr{G}$, which is compact, it follows that $\left|t_{n}\right| \rightarrow+\infty$. But there exists $a \in \mathbb{R}$ such that $t_{n} \leq v\left(x_{n}\right) \leq a$ (since $x_{n} \in \mathscr{G}$ and $v$ is continuous). Then, we deduce that $t_{n} \rightarrow+\infty$, as $n \rightarrow+\infty$. Finally, assumption (4.1) implies that $\lim _{n \rightarrow+\infty} F\left(x_{n}, t_{n}\right)=+\infty$, which yields to a contradiction with $F\left(x_{n}, t_{n}\right) \leq F(\hat{x}, v(\hat{x}))$. Then, we conclude that $E_{\alpha}$ is a compact set. Hence, problem $\left(2_{\alpha}\right)$ has at least one solution.

Step 2. We show that any solution of $\left(\mathscr{2}_{\alpha}\right)$ is regular. Let $(\tilde{x}, \tilde{t})$ be a solution of $\left(2_{\alpha}\right)$. As we have seen in the first step, we have $\sup \mathscr{2}_{\alpha} \geq 0$. Then, $v(\tilde{x}) \geq \tilde{t}$. Hence $(\tilde{x}, \tilde{t})$ is also a feasible point of $(\hat{S})$. Since $(\hat{x}, v(\hat{x}))$ solves $(\hat{S})$, it follows that $F(\hat{x}, v(\hat{x})) \leq F(\tilde{x}, \tilde{t})$. Using the fact that $(\tilde{x}, \tilde{t}) \in E_{\alpha}=\mathrm{cl} E_{\alpha}$ (where cl stands for the closure), we deduce that there exists $\left(\tilde{x}_{n}, \tilde{t}_{n}\right) \rightarrow(\tilde{x}, \tilde{t})$, as $n \rightarrow+\infty$, and $\left(\tilde{x}_{n}, \tilde{t}_{n}\right) \in E_{\alpha}$, that is,

$$
G\left(\tilde{x}_{n}\right) \leq 0, \quad F\left(\tilde{x}_{n}, \tilde{t}_{n}\right) \leq F(\hat{x}, v(\hat{x})), \quad v\left(\tilde{x}_{n}\right) \geq \tilde{t}_{n} .
$$


Since $\left(\tilde{x}_{n}, \tilde{t}_{n}\right)$ is feasible for $(\hat{S})$, it follows that $F\left(\tilde{x}_{n}, \tilde{t}_{n}\right)=F(\hat{x}, v(\hat{x}))$ (because $(\hat{x}, v(\hat{x}))$ solves $(\hat{S})$ ). On the other hand, the assumption (4.2) (see Remark 4.1) implies that there exists $(\bar{x}, \bar{t}) \in \mathbb{R} \times \mathbb{R}$, satisfying $G(\bar{x}) \leq 0, v(\bar{x})<\bar{t}$, and

$$
F(\bar{x}, \bar{t})<\inf _{\substack{(x, t) \in \mathbb{R}^{n} \times \mathbb{R} \\ G(x) \leq 0 \\ v(x) \geq t}} F(x, t)=F(\hat{x}, v(\hat{x})) .
$$

Let $\left(x_{n}, t_{n}\right)$ be the sequence defined by $\left(x_{n}, t_{n}\right)=\lambda_{n}(\bar{x}, \bar{t})+\left(1-\lambda_{n}\right)\left(\tilde{x}_{n}, \tilde{t}_{n}\right)$, with $\lambda_{n} \searrow 0^{+}$. Then, $\left(x_{n}, t_{n}\right) \rightarrow(\tilde{x}, \tilde{t})$, as $n \rightarrow+\infty$. Besides, from the convexity of the functions $G$ and $F$, we can easily check that $G\left(x_{n}\right) \leq 0$, and $F\left(x_{n}, t_{n}\right) \leq F(\hat{x}, v(\hat{x}))$. That is $(\tilde{x}, \tilde{t})$ is regular. Finally, the result follows from Proposition 2.7.

\section{References}

[1] J. P. Aubin and I. Ekeland, Applied Nonlinear Analysis, Pure and Applied Mathematics (New York), John Wiley \& Sons, New York, 1984.

[2] K. L. Hoffman, A method for globally minimizing concave functions over convex sets, Math. Program. 20 (1981), no. 1, 22-32.

[3] R. Horst, An algorithm for nonconvex programming problems, Math. Program. 10 (1976), no. 3, 312-321.

[4] R. Horst and H. Tuy, Global Optimization. Deterministic Approaches, Springer-Verlag, Berlin, 1990.

[5] Y. Ishizuka, Farkas' theorem of nonconvex type and its application to a min-max problem, J. Optim. Theory Appl. 57 (1988), no. 2, 341-354.

[6] Optimality conditions for quasi-differentiable programs with application to two-level optimization, SIAM J. Control Optim. 26 (1988), no. 6, 1388-1398.

[7] D. C. Jiang, K. L. Teo, and W. Y. Yan, A new computational method for the functional inequality constrained minimax optimization problem, Comput. Math. Appl. 33 (1997), no. 6, 53-63.

[8] M. B. Lignola and J. Morgan, Semi-continuities of marginal functions in a sequential setting, Optimization 24 (1992), no. 3-4, 241-252.

[9] R. T. Rockafellar, Convex Analysis, Princeton Mathematical Series, no. 28, Princeton University Press, New Jersey, 1970.

[10] K. Shimizu and E. Aiyoshi, Necessary conditions for min-max problems and algorithms by a relaxation procedure, IEEE Trans. Automat. Control 25 (1980), no. 1, 62-66.

[11] A new computational method for Stackelberg and min-max problems by use of a penalty method, IEEE Trans. Automat. Control 26 (1981), no. 2, 460-466.

[12] K. Shimizu and Y. Ishizuka, Optimality conditions and algorithms for parameter design problems with two-level structure, IEEE Trans. Automat. Control 30 (1985), no. 10, 986-993.

[13] K. Shimizu, Y. Ishizuka, and J. F. Bard, Nondifferentiable and Two-Level Mathematical Programming, Kluwer Academic Publisher, Massachusetts, 1997.

[14] T. Tanino and T. Ogawa, An algorithm for solving two-level convex optimization problems, Internat. J. Systems Sci. 15 (1984), no. 2, 163-174.

[15] H. Tụy, On outer approximation methods for solving concave minimization problems, Acta Math. Vietnam. 8 (1983), no. 2, 3-34.

[16] T. V. Thiều, B. T. Tâm, and V. T. Ba'n, An outer approximation method for globally minimizing a concave function over a compact convex set, Acta Math. Vietnam. 8 (1983), no. 1, 21-40, Proceeding of the IFIP Working Conference on Recent Advances in System Modelling and Optimization, Hanoi, Vietnam.

[17] H. Tuy, Concave programming under linear constraints, Soviet Math. Dokl. 5 (1964), 1437-1440. 
[18] Convex programs with an additional reverse convex constraint, J. Optim. Theory Appl. 52 (1987), no. 3, 463-486.

[19] H. Tụy and N. Q. Thái, Minimizing a concave function over a compact convex set, Acta Math. Vietnam. 8 (1983), no. 1, 13-20.

[20] H. Tuy, T. V. Thieu, and N. Q. Thai, A conical algorithm for globally minimizing a concave function over a closed convex set, Math. Oper. Res. 10 (1985), no. 3, 498-514.

Abdelmalek Aboussoror: Centre des Études Universitaires de Safi, Université Cadi Ayyad, Sidi Bouzid, P.O. Box 4162, Safi, Morocco

E-mail address: aboussororabdel@hotmail.com

Hicham Babahadda: Département de Mathématiques, Faculté des Sciences Semlalia, Université Cadi Ayyad, P.O. Box 2390, Marrakech 40000, Morocco

E-mail address: hichambabahadda@hotmail.com

Abdelatif Mansouri: Département de Mathématiques, Faculté des Sciences Semlalia, Université Cadi Ayyad, P.O. Box 2390, Marrakech 40000, Morocco

E-mail address: mansouri@ucam.ac.ma 


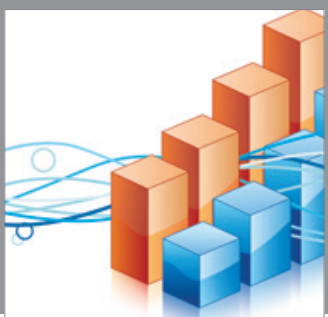

Advances in

Operations Research

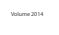

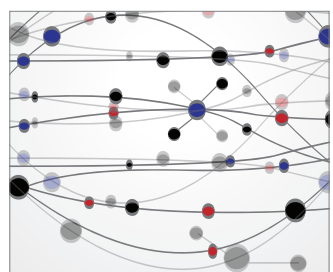

\section{The Scientific} World Journal
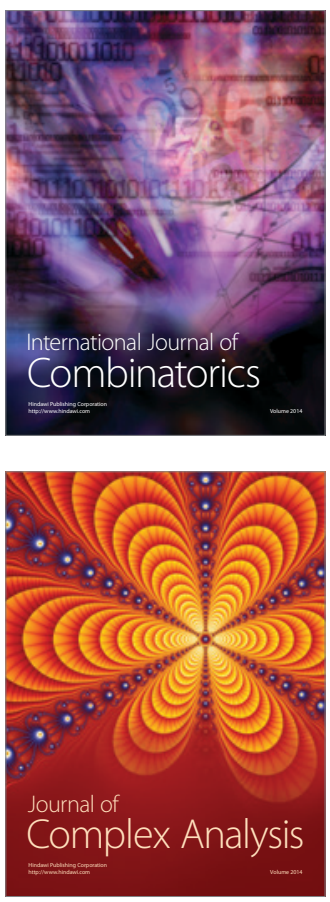

International Journal of

Mathematics and

Mathematical

Sciences
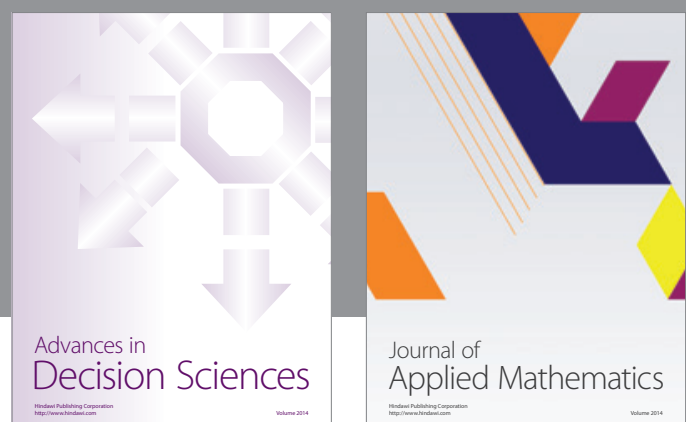

Journal of

Applied Mathematics
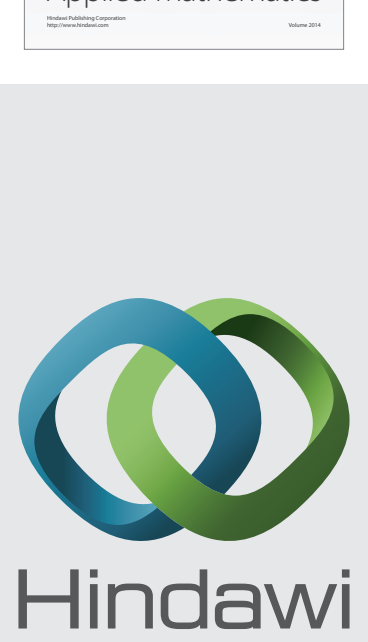

Submit your manuscripts at http://www.hindawi.com
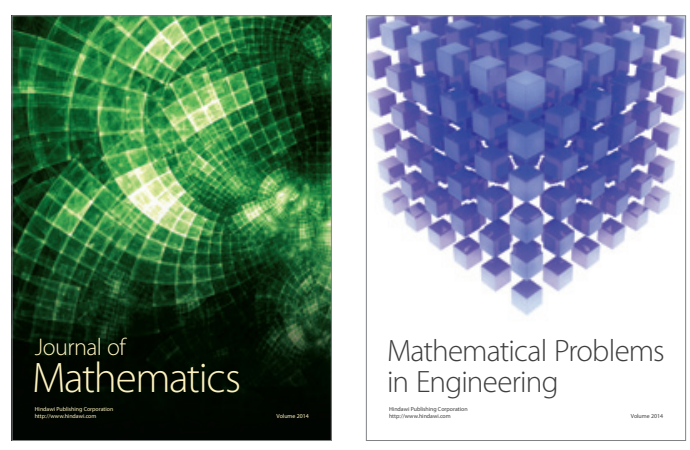

Mathematical Problems in Engineering
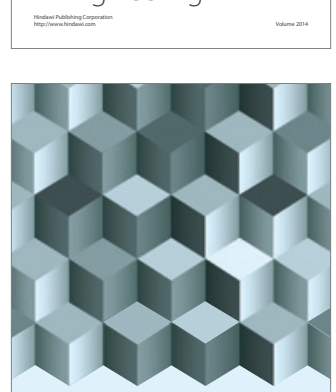

Journal of

Function Spaces
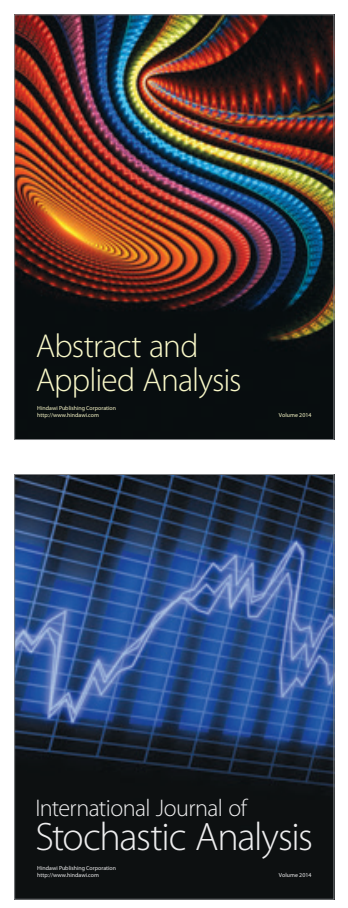

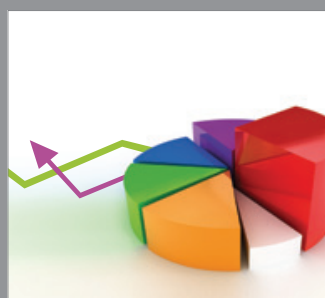

ournal of

Probability and Statistics

Promensencen
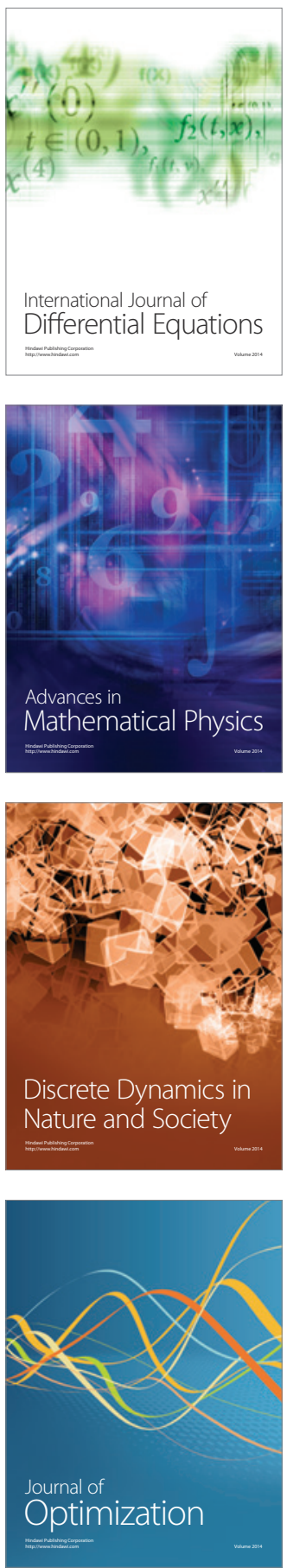